\title{
Measuring black hole masses at high redshift
}

\author{
Kelly D. Denney* \\ $\dagger$

\section{Dark Cosmology Centre, Niels Bohr Institute, Denmark}

E-mail: kelly@dark-cosmology.dk

\begin{abstract}
Understanding the connection between black holes and their host galaxies necessitates reliably measuring black hole masses across cosmic time. Reverberation mapping provides reliable direct mass measurements, but the reliability of indirect, single-epoch masses at high-z, based on black hole mass scaling relationships using the C IV emission line, has been questioned. We show that much of this seeming unreliability is due to biases that are straightforward to avoid or mitigate. In particular, we look at the effect of data quality and discuss whether or not low quality, i.e. survey, data hinder the ability to reliably estimate these masses. We also find from reverberation mapping studies that there is a 'non-variable' component of the observed single-epoch C IV profile that does not seem to be reverberating the continuum variations and is therefore likely emitted from outside the virialized broad line region. Subsequently, the estimated C IV gas velocities used for calculating the single-epoch black hole mass are biased when using the FWHM of the line, which is more sensitive to the core of the line where the non-variable component is typically strongest. After accounting for such biases by using high quality data and the line dispersion to characterize the C IV gas velocities, C IV-based masses are significantly more consistent with those based on $\mathrm{H} \beta$, with a scatter of only 0.28 dex for our sample.
\end{abstract}

Nuclei of Seyfert galaxies and QSOs - Central engine \& conditions of star formation November 6-8, 2012

Max-Planck-Insitut für Radioastronomie (MPIfR), Bonn, Germany

\footnotetext{
*Speaker.

$\dagger$ K.D. acknowledges support from the People Programme (Marie Curie Actions) of the European Union's Seventh Framework Programme FP7/2007-2013/ under REA grant agreement no. 300553 and from HST grant HST-AR-12149 awarded by the Space Telescope Science Institute, which is operated by the Association of Universities for Research in Astronomy, Inc., for NASA, under contract NAS5-26555. The Dark Cosmology Centre is funded by the Danish National Research Foundation
} 


\section{Introduction}

Super-massive black holes (BHs) are believed to be ubiquitous in the centers of massive galaxies. Understanding their existence and growth and the connection these BHs have to the evolution of their host galaxies requires knowledge of $\mathrm{BH}$ demographics across cosmic time. At present, $\mathrm{BH}$ masses at high redshift are principally estimated indirectly with single-epoch (SE) BH mass scaling relationships applicable to Type 1, or broad-line, Active Galactic Nuclei (AGNs). These SE mass estimates use the AGN continuum luminosity as a proxy for the radius of gravitationally-bound, broad line-emitting gas (Bentz et al. 2009), and the broad line width as a measure of the broad line region (BLR) gas velocity, $\Delta V$, so that the virial mass is estimated by $M \propto L^{\alpha} \Delta V^{2}$. Direct measurements are typically not available because these methods rely on either spatially resolving the $\mathrm{BH}$ gravitational radius of influence (see, e.g., Ferrarese \& Ford 2005, and references therein), which is not possible outside the local universe, or temporally resolving the reverberation time delay between the AGN continuum emission and that of broad emission lines (e.g., Peterson et al. 2004), which, though not fundamentally restricted to the local AGN population, has remained nearly so thus far due to the logistical difficulty of scheduling the required observations when time dilation of the reverberation signal becomes significant.

At redshifts $z \gtrsim 2$, the only broad AGN emission line with a calibrated mass scaling relation in the easily-accessible optical observing window is C IV $\lambda 1549$, making it the primary emission line available for high- $z \mathrm{BH}$ and galaxy evolution studies based on ground-based optical data. Many past studies have questioned the reliability of C IV as a virial mass indicator (e.g., Baskin \& Laor 2005; Sulentic et al. 2007; Netzer et al. 2007, hereafter N07; Shen \& Liu 2012; Trakhtenbrot \& Netzer 2012) due to a general inconsistency, in the form of scatter and or mean offsets, between $\mathrm{C}$ IV- and $\mathrm{H} \beta$-based SE masses. Nonetheless, available reverberation mapping (RM) results for $\mathrm{C}$ IV yield consistent $\mathrm{BH}$ mass results with those of the other emission lines observed in the same objects (Peterson \& Wandel 2000; Peterson et al. 2004), so any issues are restricted to SE estimates using $\mathrm{CIV}$, rather than $\mathrm{CIV}$ in general. Because of the consistency between the RM-based mass results for $\mathrm{CIV}$ and $\mathrm{H} \beta$, we postulated that the in-consistency between SE C IV and $\mathrm{H} \beta$ masses is more likely due to an observational or systematic bias than an intrinsic limitation of C IV, and set out to investigate this possibility. The results of this investigation are presented in the following two sections and have been drawn from the work of Denney et al. (2013) and Denney (2012).

\section{The effects of data quality on C IV mass estimates}

We noticed that the majority of studies questioning the reliability of C IV-based SE mass estimates utilized typically low-quality, i.e., low $S / N$ survey data, from which to draw their conclusions, while many proponents of C IV used, typically, high $S / N$ spectra from HST or smaller, proprietary samples. We therefore investigated the direct effects that data quality can have on C IVbased SE mass estimates by (1) re-observing several SDSS quasars, for which N07 estimate the $\mathrm{C}$ IV masses from the SDSS spectra and the $\mathrm{H} \beta$ masses from Gemini Near-Infrared Spectrograph observations, and directly comparing the low- and high-quality C IV profiles and masses, and (2) collecting a relatively large ( 47 objects) sample of high quality $\mathrm{C}$ IV spectra for objects that also have $\mathrm{H} \beta$-based mass measurements to use for comparing $\mathrm{C}$ IV and $\mathrm{H} \beta$ masses. 
Figure 1 shows three example spectra of the SDSS sample from N07 for which we obtained new, high $S / N$ spectra of the C IV emission-line region with the first of the Multi-Object Double Spectrographs (MODS1; Pogge et al. 2010) on the Large Binocular Telescope. We have fit each line profile with a linearly interpolated continuum fit between continuum regions $\sim 1450 \AA$ and $\sim 1700 \AA$ and one or more sixth-order Gauss Hermite polynomials as a means to best reproduce the underlying C IV profile. Even a simple qualitative comparison of the MODS1 spectra to the SDSS spectra demonstrate that significant improvements are afforded by the increased data quality in the MODS 1 specta by ability to accurately define the intrinsic C IV emission-line profile and underlying continuum and to accurately identify the prevalence, location, and strength of absorption.
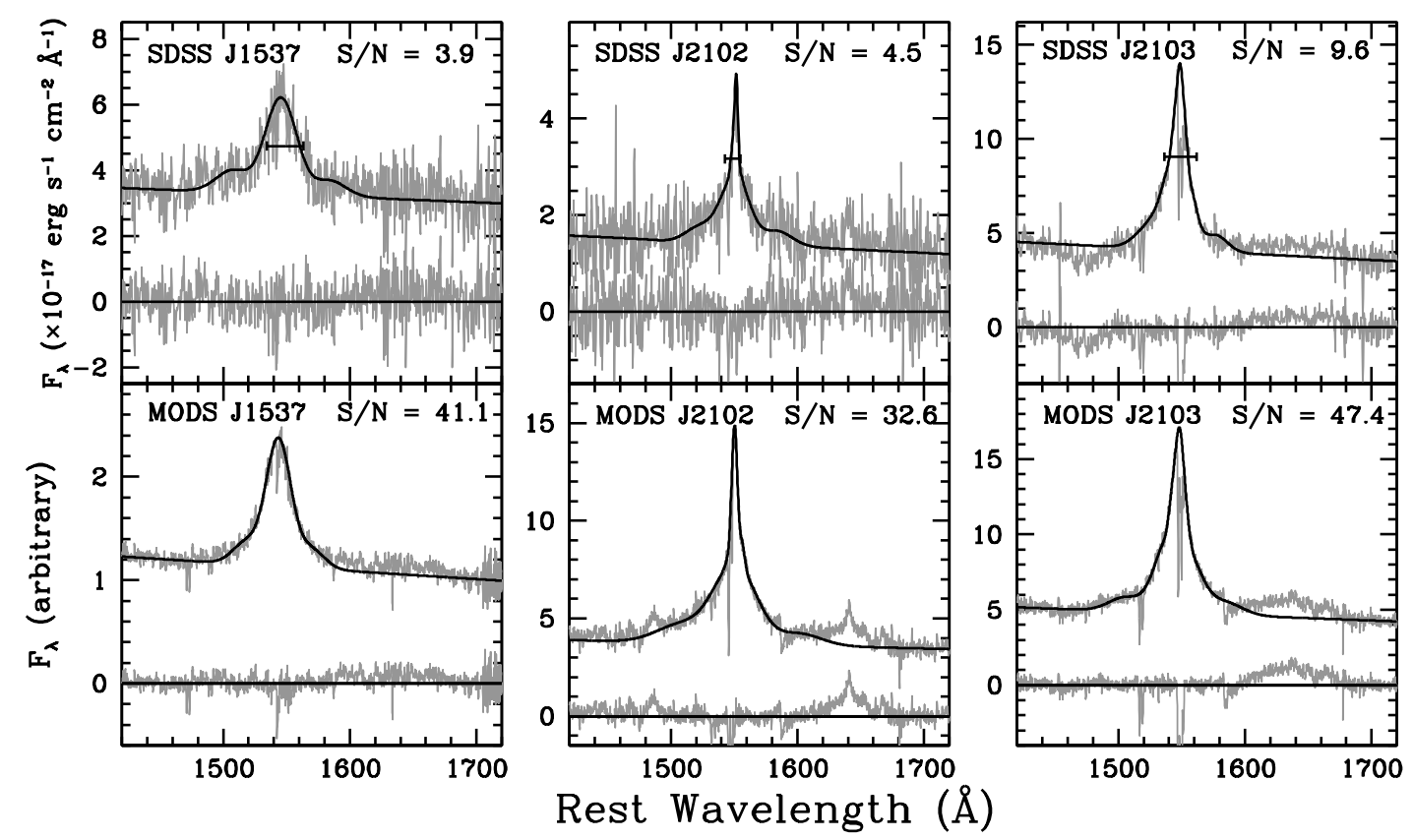

Figure 1: Example spectra (gray), composite continuum plus C IV profile fits (solid black), and residuals for some of N07 sample objects observed with SDSS (top) and MODS1 (bottom). The $S / N$ of each spectrum measured per resolution element in the continuum is given in the top corner of each panel. The black bar across the SDSS C IV profile (top) shows the FWHM velocity width reported for the C IV emission line in this object by N07. This figure is taken from Denney et al. (2013).

Figure 2 compares the $\mathrm{C}$ IV and $\mathrm{H} \beta$ masses derived for our full sample of 47 objects. The left panel shows this comparison for C IV masses determined using the FWHM, and right panel shows the same comparison, but using the line dispersion, $\sigma_{l}$, the second moment of the C IV line profile. These results demonstrate that even with high quality data, the scatter between $\mathrm{H} \beta$ masses and FWHM-based C IV masses is just as large as previous comparisons using a heterogeneous-quality sample or strictly survey-quality data. On the other hand, when characterizing the C IV line profile with $\sigma_{l}$ in only high-quality data, the scatter between the C IV and $\mathrm{H} \beta$ masses is significantly reduced (only $0.28 \mathrm{dex}$ ). This demonstrates that reliable C IV SE masses can be estimated when using (1) high quality data, AND (2) $\sigma_{l}$ to characterize the C IV velocity width. 

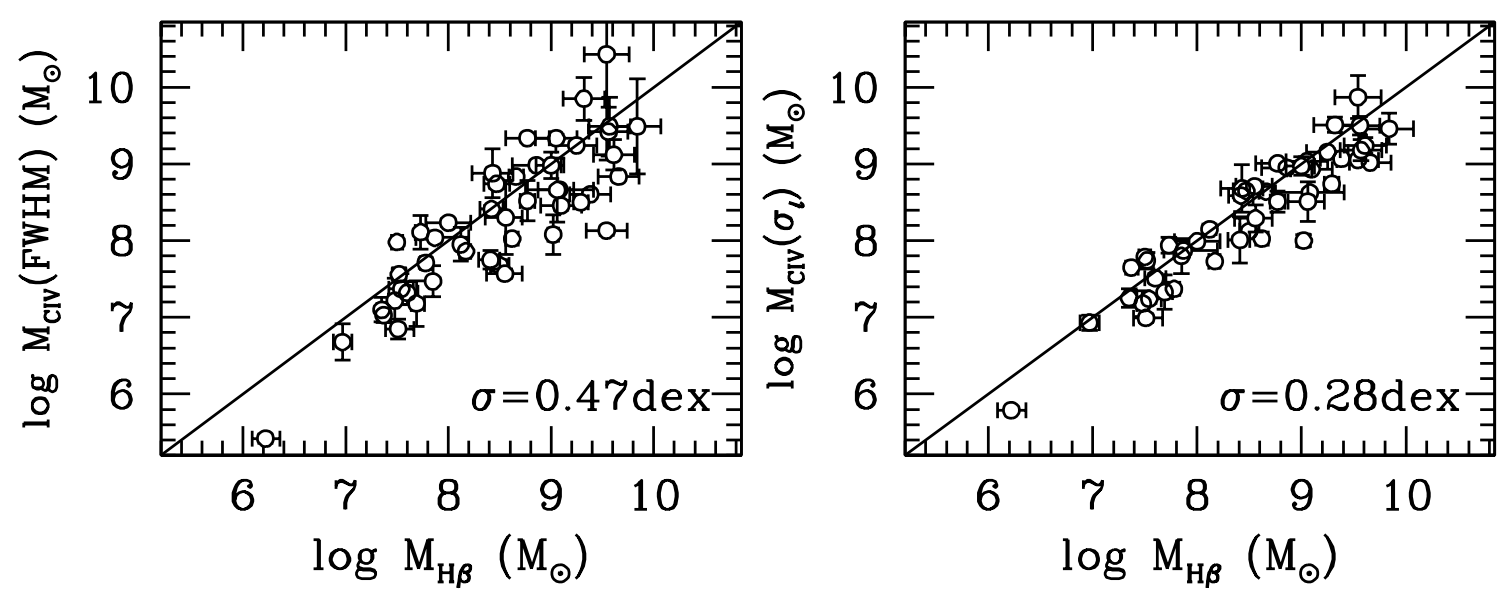

Figure 2: Comparison between $\mathrm{H} \beta$ masses and $\mathrm{C}$ IV masses estimated from our sample of 47 high quality spectra. The left (right) panel shows C IV masses based on the FWHM $\left(\sigma_{l}\right)$. The solid line shows where the $\mathrm{C}$ IV and $\mathrm{H} \beta$ masses are equal. The scatter, i.e., the standard deviation about the mean of the C IV-to-H $\beta$ mass residuals, $\sigma$, is shown in the bottom right of each panel. Adapted from Denney et al. (2013).

\section{Systematic bias to the FWHM of C IV due to non-variable emission}

Since improved data quality was not the cause of the large scatter observed between FWHMbased C IV masses and $\mathrm{H} \beta$ masses, we asked the question, "What is?" To address this question, we returned to the reverberation mapping sample of objects that also show consistency between $\mathrm{C} I V$ and $\mathrm{H} \beta$-based masses and looked specifically at differences between the mean profile, which is essentially equivalent to a SE profile, and the rms profile typically used to derive an RM-based mass. Figure 3 compares these two profiles for some of the objects that have C IV RM results. Interestingly, in all cases the rms profiles are missing an emission component seen in the mean profile. In other words, there is a C IV emission component that does not vary and is not reverberating the signal from the continuum. Likely, then, the gas responsible for this emission is not part of the same distribution of gas expected to be from the BLR and whose motions are under the gravitational influence of the $\mathrm{BH}$. These gas velocities should therefore not be taken into account when estimating a SE virial BH mass. C IV mass estimates based on the FWHM of the line can therefore be significantly biased by the presence of this non-variable component that typically arises in the core of the C IV profile, to which the FWHM is most sensitive.

Unfortunately, this non-variable component does not approximate the non-variable narrow emission component typically seen superposed with the broad Balmer emission lines, and arising in an extended, [O III] $\lambda \lambda 4959,5007$-like narrow-line region (NLR). Thus, its contribution cannot be simply subtracted by using another narrow emission line as a template. Instead, the non-variable emission profile varies between objects; however, the contamination of this emission component to the FWHM-based C IV mass can be estimated from the overall shape of the C IV emission line. The left panel of Figure 4 shows that objects with typically more 'peaky' ('boxy') C IV profiles tend to have under- (over-) estimated masses compared to $\mathrm{H} \beta$. This correlation makes it possible to derive a potential correction for the FWHM-based masses (see Denney 2012 and Denney et al. 2013 for details). Conversely, there is no significant bias introduced to the $\sigma_{l}$-based C IV masses from the 

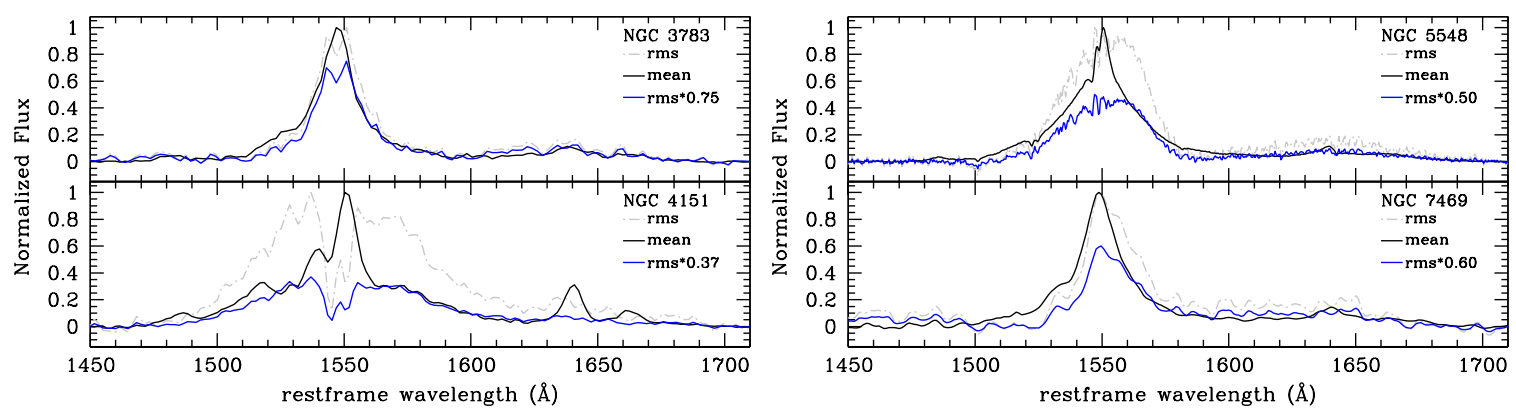

Figure 3: Examples of continuum-subtracted mean (black) and rms (gray dot-dashed) spectra from the C IV RM sample that have been normalized to the C IV peak flux. The blue curves show the same rms spectra scaled by an arbitrary factor to approximately match the red wing flux between each rms and mean spectrum. Adapted from Denney (2012).

non-variable component, as evidenced by the lack of a correlation in the right panel of Figure 4. This explains the much smaller scatter seen in Figure 2 for $\sigma_{l}$-based C IV masses compared to $\mathrm{H} \beta$.
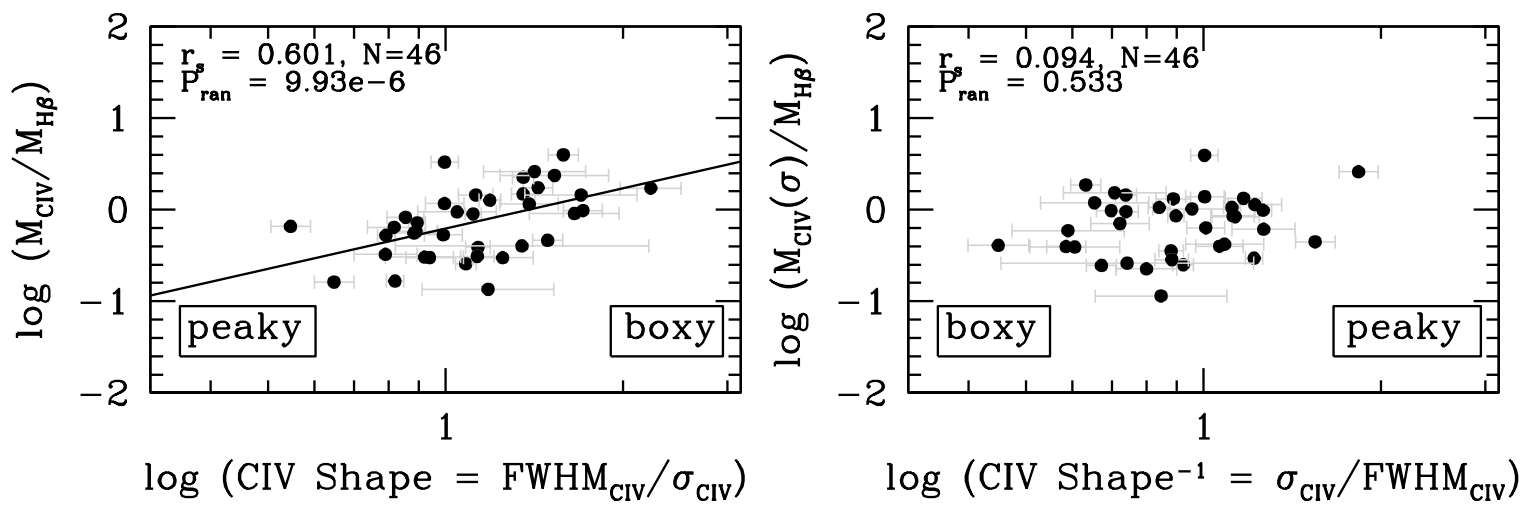

Figure 4: Left: Comparison between $\mathrm{C}$ IV and $\mathrm{H} \beta$ mass residuals, calculated using FWHM, and the shape of the C IV line, $S=$ FWHM $/ \sigma_{l}$. The solid line shows the best fit correlation. The Spearman rank order coefficient, $r_{\mathrm{s}}$, the number of objects included in the fit, $N$, and the probability that the correlation is found by chance, $P_{\text {ran }}$, are given in the top right corner. Right: Same except the C IV masses were calculated using $\sigma_{l}$, and the resulting residuals with the $\mathrm{H} \beta$ masses are compared to $S^{-1}$ so to have the same dependence on the C IV width characterization used in the mass estimates. Adapted from Denney (2012).

\section{Conclusion}

Reliably determining C IV BH masses is of significant interest to the community, since these masses are essential for high redshift studies of the cosmic evolution and growth of BHs and their connection to galaxy evolution through, e.g., feedback. Despite common claims that SE CIV masses cannot be reliably estimated, based on a comparison to $\mathrm{H} \beta$ masses that are better calibrated locally with RM studies, we have shown here that they can, in fact, be reliably estimated - to within a relative uncertainty of $<0.3 \mathrm{dex}$. The requirements are simply to characterize the BLR 
velocity from the line dispersion of the C IV profile and to do so with high-quality data $(S / N>10$ pixel $^{-1}$ measured in the continuum). Furthermore, we have determined that much of the scatter between the $\mathrm{H} \beta$ masses and SE CIV masses based on the FWHM of the CIV line is due to the presence of a non-variable emission component in the SE C IV profile that is likely not arising from the virialized BLR. It therefore significantly contaminates the measurement of the gas velocities relevant for calculating a virial BH mass when the FWHM of the C IV line is used.

\section{References}

[1] M. C. Bentz, B. M. Peterson, H. Netzer, R. W. Pogge, and M. Vestergaard, The RadiusLuminosity Relationship for Active Galactic Nuclei: The Effect of Host-Galaxy Starlight on Luminosity Measurements. II. The Full Sample of Reverberation-Mapped AGNs, ApJ 697 (May, 2009) 160-181, [0812.2283].

[2] L. Ferrarese and H. Ford, Supermassive Black Holes in Galactic Nuclei: Past, Present and Future Research, Space Science Reviews 116 (Feb., 2005) 523-624, [arXiv:astro-ph/0411247].

[3] B. M. Peterson et al., Central Masses and Broad-Line Region Sizes of Active Galactic Nuclei. II. A Homogeneous Analysis of a Large Reverberation-Mapping Database, ApJ 613 (Oct., 2004) 682-699.

[4] A. Baskin and A. Laor, What controls the CIV line profile in active galactic nuclei?, MNRAS 356 (Jan., 2005) 1029-1044, [arXiv: astro-ph/ 0409196$].$

[5] J. W. Sulentic, R. Bachev, P. Marziani, C. A. Negrete, and D. Dultzin, C IV $\lambda 1549$ as an Eigenvector 1 Parameter for Active Galactic Nuclei, ApJ 666 (Sept., 2007) 757-777, [0705.1895].

[6] H. Netzer, P. Lira, B. Trakhtenbrot, O. Shemmer, and I. Cury, Black Hole Mass and Growth Rate at High Redshift, ApJ 671 (Dec., 2007) 1256-1263, [0 708 .3787].

[7] Y. Shen and X. Liu, Comparing Single-epoch Virial Black Hole Mass Estimators for Luminous Quasars, ApJ 753 (July, 2012) 125, [1203.0601].

[8] B. Trakhtenbrot and H. Netzer, Black Hole Growth to $z=2$ - I: Improved Virial Methods for Measuring M_BH and L/L_Edd, MNRAS 427 (Dec., 2012) 3081, [1209.1096].

[9] B. M. Peterson and A. Wandel, Evidence for Supermassive Black Holes in Active Galactic Nuclei from Emission-Line Reverberation, ApJL 540 (Sept., 2000) L13-L16, [arXiv:astro-ph/0007147].

[10] K. D. Denney, Are Outflows Biasing Single-Epoch CIV Black Hole Mass Estimates?, ApJ 759 (Oct., 2012) 44, [1208.3465].

[11] K. D. Denney, R. W. Pogge, R. J. Assef, C. S. Kochanek, B. M. Peterson, and M. Vestergaard, CIV Line-width Anomalies: The Perils of Low S/N Spectra, to be submitted to ApJ (Feb., 2013). 